\title{
The Dependence of Properties of the Electroplated Coatings on the Spectrum of the Variable Components of the Current (Voltage)
}

\author{
V. F. Gologan, Zh. I. Bobanova, S. Kh. Ivashku, S. P. Sidelnikova \\ Institute of the Applied Physics of the Moldova Academy of Sciences, ul. Akademicheskaya, Chisinau, \\ MD-2028, Republic of Moldova \\ *Corresponding Author: V. F. Gologan, Institute of the Applied Physics of the Moldova Academy of \\ Sciences, ul. Akademicheskaya, Chisinau, MD-2028, Republic of Moldova
}

Received Date: 13-06-2017

Accepted Date: 22-06-2017

Published Date: 27-06-2017

\begin{abstract}
The results of the studies devoted to the effect of the parameters of the inductance-capacitance device on the process of deposition of galvanic coatings, structure and properties of the coatings are discussed. The form of the current of the power source and the values of the inductunce and the device capacity are shown to affect substantially the formation of the spectrum of the variable components in the galvanic circuit, which, as a result changes the cathode polarization, as well as the structure and properties of the coating. In the presence of a spectrum of the variable components the favourable conditions arise that create a polyfrequency resonance.
\end{abstract}

Keywords: Inductance-capacitance device, spectrum, structure, coating

\section{INTRODUCTION}

In the electrochemical processes, the phenomena are known to appear, which induce the current (voltage) pulsation [1-4]. These fluctuations reflect the electrode reactions caused by the elementary processes on the electrodes. Thus, if the stationary corrosion transforms into the nonstationary one, i.e., if we have to do with the corrosion localization (the pitting formation), it can be identified by the method of the "noises" record, using certain techniques and special equipment and apparatuses [1].

The above method of electrochemical noises can also be used for the diagnosis of organic electrolytes of accumulators with a metal lithium electrode [5], as well as to determine the degree of a source discharge.

V. A. Tyagai found that a slow discharge, fluctuations of reagents in the near-cathode region, the rate of a chemical reaction, photoexitation, intensive gas release (hydrogen, oxygen) on the electrodes, circuit resistance, etc., can be the sources of fluctuations in the electrical circuit rectifier-bath. Therefore, the values of the variable components of current (voltage), the same as the polarization curves, reflect the electrochemical kinetics of deposition of galvanic coatings [4]. The aforementioned suggests that along with the composition and temperature of the electrolyte, density and form of the current, etc., we found another possibility to affect the process of deposition of galvanic coatings by changing the characteristics of the variable components (VC) of the current (voltage). For this purpose it was suggested to use an inductance-capacitance device (ICD), that is connected in series (or embedded) with the main power source (PS), by changing the parameters (inductance L and capacity $\mathrm{C}$ ) of which we can affect substantially the value and frequency of the $\mathrm{VC}$ [6]. The inductance and capacity are generally connected in parallel; however, in the case of a switching power supply their connection in series is possible [7, 8].

Over the recent years we have performed numerous studies using the ICD, and, therefore, it is worthwhile to generalize the obtained results and to develop recommendations for their application.

\section{THE EFFECT OF THE ICD PARAMETERS ON The Cathode Potential and VC SPECTRUM}

The cathode potential was registered during the deposition of the copper, nickel, chromium and 
The Dependence of Properties of the Electroplated Coatings on the Spectrum of the Variable Components of the Current (Voltage)

other types of coatings on the $1 \mathrm{~cm}^{2}$ platinum plate in a standard cell ES-2 and a stepwise regulation of the current with an exposure for 30 $\mathrm{s}$ at each value which was necessary for stabilization of the potential [9]. The current value was determined by means of a resistance box and was measured using an M209 voltammeter. A saturated calomel electrode was used as a reference electrode. The cathode potential was measured using a V7-27A/1 voltammeter. The form of the current was registered with S1-55 and GDS-1072 oscillographs, and the variable components of the current in the circuit power source-bath were studied using a SK4-56 frequency analyzer. Inductance and capacity were measured with a LCR-200 apparatus.

The inductance was formed mainly by means of two similar impedance coils $\mathrm{L}=5 \mathrm{H}$, whose choke coils were connected in series and in parallel. The necessary capacity was ensured by a parallel connection of electrolytic condensers,

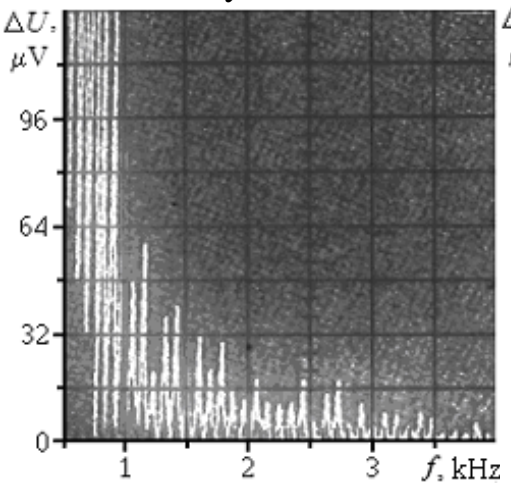

a and mainly in the experiments the condenser capacity was $\mathrm{C}=2200 \mathrm{mkF}$. For the deposition of the coating we used, as a rule, a single-phase, three-phase, six-phase and 60W switching power sources, as well as a lead accumulator [10-15].

The analysis of the results of the studies showed that the current pulsations of the power sources affect the VC spectrum in the circuit without the connection of the electrolyzer. With an increase in the pulsations of the main current there was an increase both in their value and frequency.

These features of construction of the power sources affect substantially the VC spectrum also after the connection with bath during the deposition of copper from the sulphate electrolyte $\left(\mathrm{CuSO}_{4}-250 \mathrm{~g} / \mathrm{l}, \mathrm{H}_{2} \mathrm{SO}_{4}-50 \mathrm{~g} / \mathrm{l}, \mathrm{t}_{\mathrm{el}}-\right.$ $20^{\circ} \mathrm{C}, \mathrm{i}_{\mathrm{k}}-0,2 \mathrm{\kappa A} / \mathrm{m}^{2}, \mathrm{I}=100 \mathrm{~mA}$ ) (Fig. 1, samples a, b).

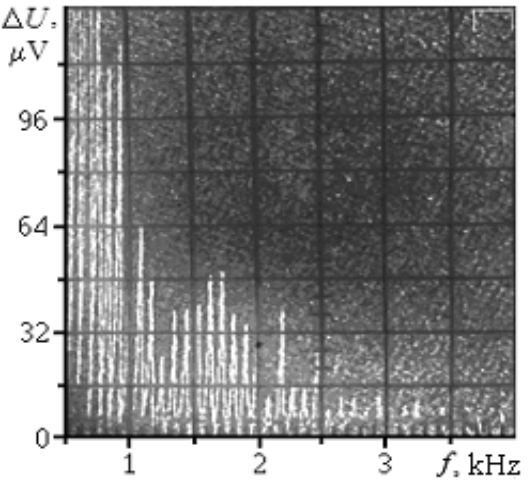

b

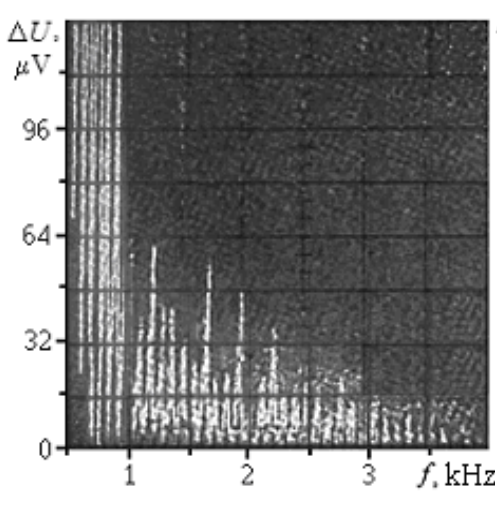

C

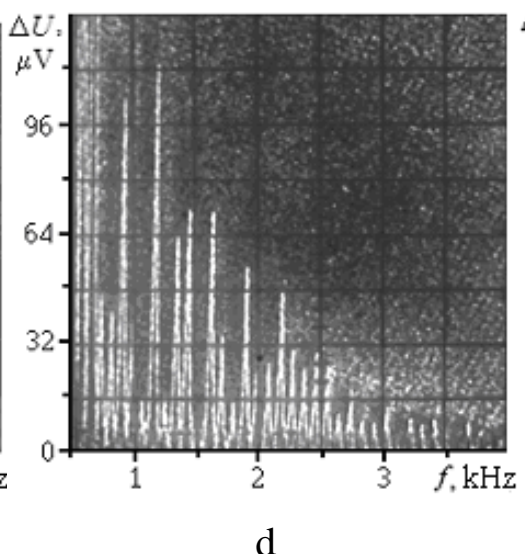

d

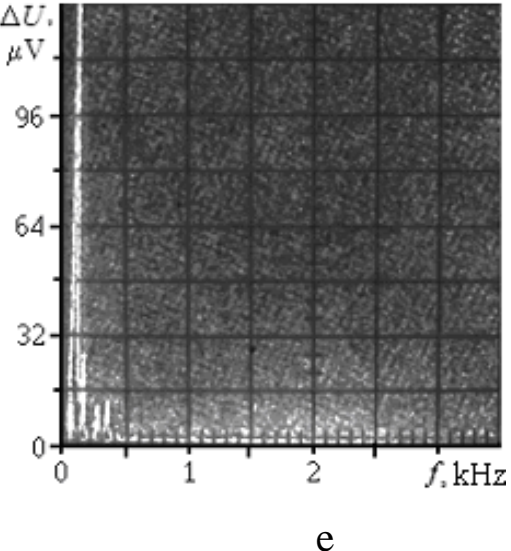

e

Fig1. The VC spectra $\left(I=100 \mathrm{MA}, 0,2 \kappa \mathrm{A} / \mathrm{M}^{2}\right)$ during the copper deposition from the sulphate electrolyte $\left(\mathrm{CuSO}_{4} \cdot 5 \mathrm{H}_{2} \mathrm{O}-200 \mathrm{~g} / \mathrm{l}, \mathrm{H}_{2} \mathrm{SO}_{4}-50 \mathrm{~g} / \mathrm{l}, t_{e l}-20^{\circ} \mathrm{C}\right):$ a - without the ICD connection and $a$ bath; $b-$ with the ICD connection $\left(L_{o p t}=10 \mathrm{H}, C_{o p t}=17600 \mathrm{mkF}\right)$ without the bath. With copper deposition; $c-$ without the ICD connection; $d$ - with the ICD connection $\left(L_{o p t}=10 \mathrm{H}, C_{o p t}=17500 \mathrm{mkF}\right) ; e-$ only with the inductance connection $(L=10 \mathrm{H})$.

Changing the ICD parameters we can affect the variable components in the circuit. Varying the inductance, we align the initial form of the current with the VC being actually absent. Connecting capacities parellel to inductance, we can observe compensation of the inductance and 
The Dependence of Properties of the Electroplated Coatings on the Spectrum of the Variable Components of the Current (Voltage)

recovery of the initial form of the current. However, in this case, the VC values increase and the spectrum increases towards the higher frequencies (Fig. 1, samples c, d).
The polarization curves show that the connection of only L causes a shift of the cathode potential towards the negative region (Fig. 2).

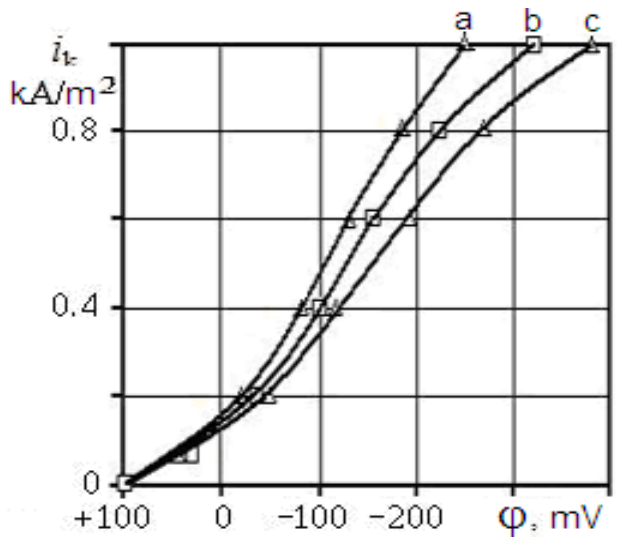

Fig2. Polarization curves during the copper deposition (with a single-phase power supply) from sulphate electrolyte: $a$ - with the ICD connection $\left(L_{o p t}=10 \mathrm{H}, C_{o p t}=17500 \mathrm{mkF}\right) ; b$ - without the ICD connection; $c-$ only with the inductance connection $(L=10 \mathrm{H})$.

The cathode potential became more positive with the capacity being connected (compared to the initial value (without the ICD). The VC spectrum changed most when the inductance $\left(\mathrm{L}_{\mathrm{opt}}\right)$ totally aligned the initial form of the current and the capacity $\left(\mathrm{C}_{\mathrm{opt}}\right)$ ensured the recovery of its initial pulsations. Therefore, the VC spectrum is substantially affected by the initial form of the current of the power supply.

This inference is vividly supported in the tests using a switching power supply with a more developed VC spectrum and the variable components with a frequency up to $85 \mathrm{kHz}$ during the deposition of copper and nickel [14, 15].

In the case of using the accumulator as a power supply, it is determined that its initial VC values are in the regions of the background noise of the measuring apparatus. The VC spectrum was determined during the connection of the bath, which was obviously caused by the electrochemical process during the chromium deposition from the standard electrolyte $\left(\mathrm{CrO}_{3}-\right.$ $250 \mathrm{~g} / 1, \mathrm{H}_{2} \mathrm{SO}_{4}-2,5 \mathrm{~g} / \mathrm{l}, \mathrm{t}_{\mathrm{el}}-55^{\circ} \mathrm{C}, \mathrm{i}_{\mathrm{k}}-5,5 \mathrm{kA} / \mathrm{m}^{2}, \mathrm{I}$ $=25 \mathrm{~mA}, \mathrm{C}_{\mathrm{opt}}=0,01 \mathrm{~F}, \mathrm{~L}_{\mathrm{opt}}=0,09 \mathrm{mH}$ ) (Fig. 3).

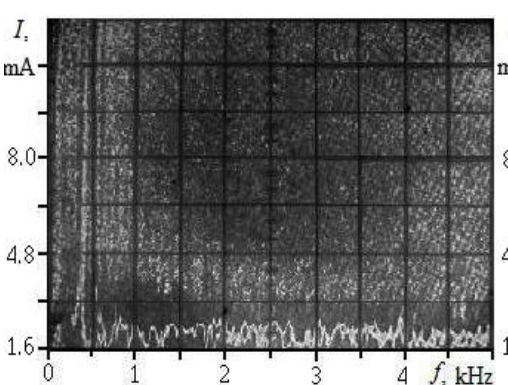

a

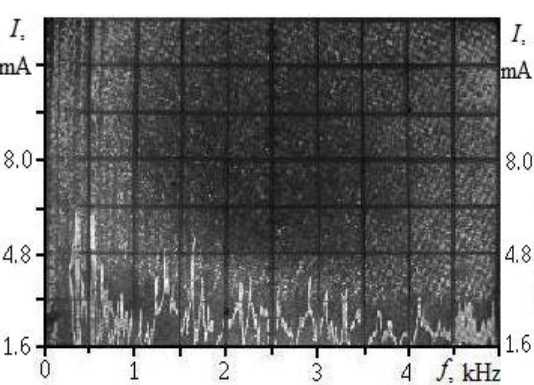

b

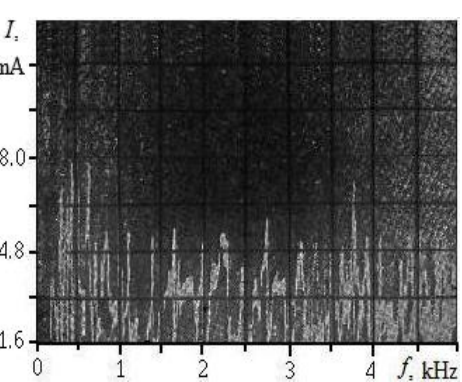

c

Fig3. The VC spectra during the chromium deposition $\left(\mathrm{CrO}_{3}-250 \mathrm{~g} / \mathrm{l}, \mathrm{H}_{2} \mathrm{SO}_{4}-2,5 \mathrm{~g} / \mathrm{l}, t_{e l}=55^{0} \mathrm{C} ; \mathrm{I}=25 \mathrm{~A}\right.$, from the accumulator: $a$ - without the bath connection; $b$ - with the bath connection; $c$ - with the ICD connection $\left(L_{\text {opt }}=0,09 \mathrm{mH}, C_{\text {opt }}=0,01 F\right) ; b-c$ подключением ванны; $c-$ с подключением ICD $\left(L_{\text {opt }}=0,09 \mathrm{mH}\right.$, $\left.C_{\text {opt }}=0,01 F\right)$.

The same as in the earlier tests, the ICD connection affects markedly the initial VC spectrum, and at the optimal values of the parameters $\left(\mathrm{L}_{\mathrm{opt}}, \mathrm{C}_{\mathrm{opt}}\right)$ its components aligned their values up to the frequency $8 \mathrm{kHz}$.

In the process of the deposition of chromium coatings from tetrachromic electrolyte $\left(\mathrm{CrO}_{3}-\right.$
$400 \mathrm{~g} / \mathrm{l}, \mathrm{NaOH}-60 \mathrm{~g} / \mathrm{l} ; \mathrm{H}_{2} \mathrm{SO}_{4}-2,5 \mathrm{~g} / \mathrm{l}$, sugar $1 \mathrm{~g} / 1$, the electrolyte temperature was $20-22^{\circ} \mathrm{C}$, $\mathrm{i}_{\mathrm{k}}-2,0 \mathrm{kA} / \mathrm{m}^{2}, \mathrm{I}-2 \mathrm{~A}$ ) and using a 3-phase power supply [13], the ICD effect was vividly apparent on certain components of the spectrum (Fig. 4). 
The Dependence of Properties of the Electroplated Coatings on the Spectrum of the Variable Components of the Current (Voltage)

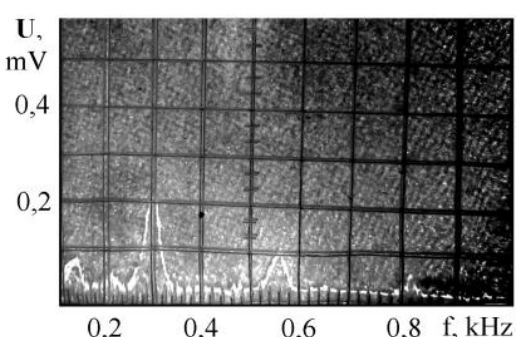

a

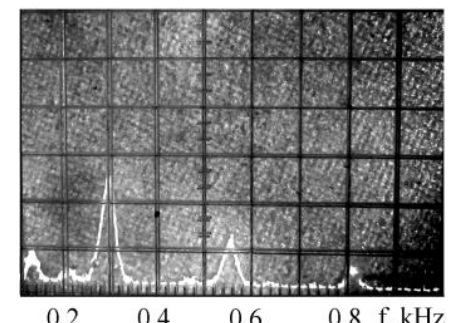

b

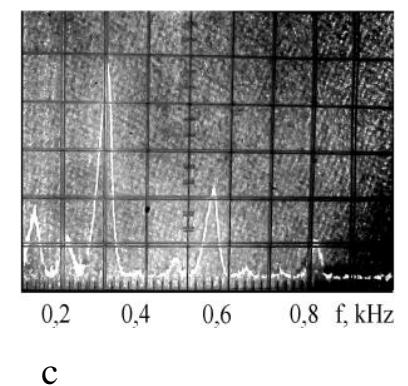

c

Fig4. The VC spectra (sensitivity 0,8mV) during chromium depositionnpu using 3-phase power supply from tetrachromatic electrolyte $\left(\mathrm{CrO}_{3}-400 \mathrm{~g} / \mathrm{l}, \mathrm{NaOH}-60 \mathrm{~g} / \mathrm{l}, \mathrm{H}_{2} \mathrm{SO}_{4}-2,5 \mathrm{~g} / \mathrm{l}\right.$, sugar $-1 \mathrm{~g} / \mathrm{l}, i_{k}-2,0 \mathrm{\kappa A} / \mathrm{m}^{2}, \mathrm{I}=100 \mathrm{~A}$, $\left.t_{\text {opt }}=20-22^{\circ} \mathrm{C}\right): a$-only from PS; $b$ - with the bath connection; $c$ - with the ICD connection $\left(L_{o p t}=5 H\right.$,

$C_{\text {opt }}=17600 \mathrm{mkF}$ ).

This type of power sources $(60 \mathrm{~W})$ were selected for the purpose of conducting the tests during recording the polarization curves, spectra and coating deposition on the samples, ment for other kinds of tests at currents close in value. With an increase in the $\mathrm{VC}$ current the efficiency of the ICD application also increases, enhances and, simultaneously, decreases the necessity of using high inductance [12], and the voltage drop in the device is up to $5 \%$ from the total value.

The change in the kinetics of the coating deposition with the ICD connection affected the current efficiency. Thus, during the deposition of chromium coatings using a 3-phase power supply in the standard electrolyte $\left(\mathrm{i}_{\mathrm{k}}-5,5 \mathrm{kA} / \mathrm{m}^{2}\right.$, $\mathrm{I}-25 \mathrm{~A})$ and at $\mathrm{L}_{\text {opt }}=0,119 \mathrm{mH}, \mathrm{C}_{\text {opt }}=0,024 \mathrm{mF}$, the current efficiency increases by 1.5-1.6 times,

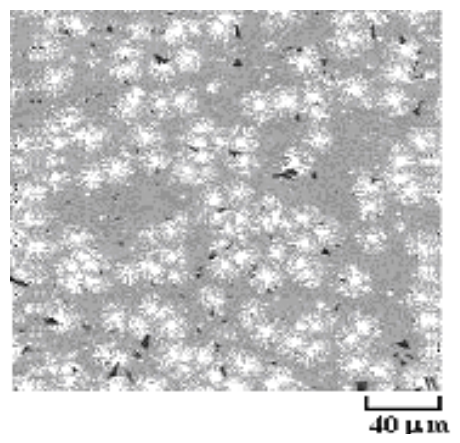

a

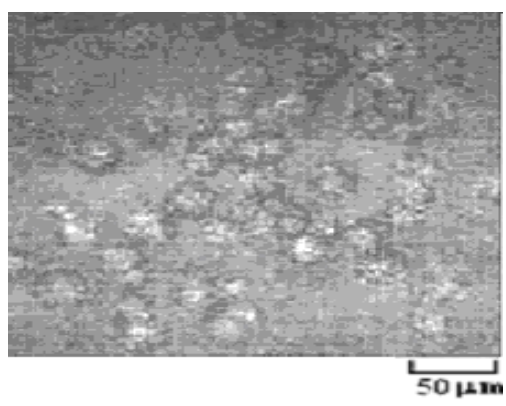

$\mathrm{c}$ which attests to a substantial change in the electrochemical process [12].

\section{The ICD EFfect ON THE STruCture AND Properties of The CoAtings}

The numerous studies showed that the ICD parameters affect substantially the morphology, structure and properties of the coatings. In the process of copper deposition (a single-phase power supply) from the sulphate electrolyte $\left(t_{e l}\right.$ $-20^{\circ} \mathrm{C}, \mathrm{i}_{\mathrm{k}}=0,2 \mathrm{\kappa A} / \mathrm{m}^{2}, \mathrm{i}=63 \mathrm{~mA}$ ) for $15 \mathrm{~s}$ on a molybdenum monocrystal that was polished using chromium oxide, it was determined [16] that first a nucleation center was formed and then (radially) dendrites were growing, 0.15$0.5 \mathrm{mkm}$ in the cross section and $10 \mathrm{mkm}$ long (Fig. 5, samples a, b).

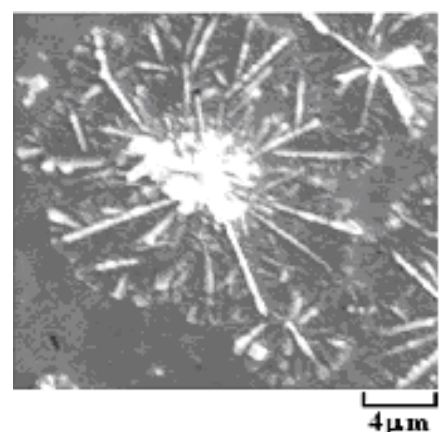

b

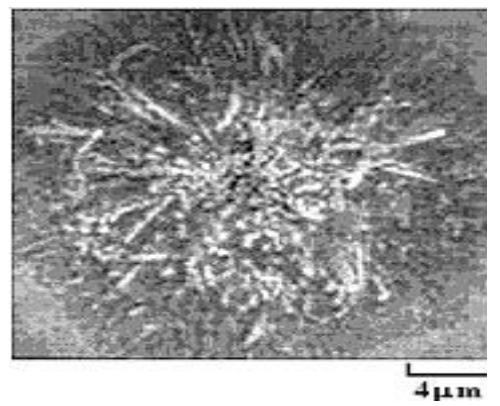

$\mathrm{d}$

Fig5. Morphology of copper deposited from sulphate electrolyte on the surface of the monocrystal for $15 \mathrm{~s}$ : $a, b$-without the ICD connection; $c, d$-with the ICD connection $(L=10 \mathrm{H}, C=17600 \mathrm{mkF})$. 
The Dependence of Properties of the Electroplated Coatings on the Spectrum of the Variable Components of the Current (Voltage)

The ICD connection $(\mathrm{L}=10 \mathrm{H}, \mathrm{C}=17600 \mathrm{mkF})$ changed the sediment formation (Fig. 5, samples c, d). The copper deposition during the same period of time resulted in the formation of a continious coating, which occurred over $25-30 \%$ of the cathode area, and there were large "spots" with a diameter of $30-40 \mathrm{mkm}$ over the remaining surface of the electrode with a pronounced centers. The spots differed by their external

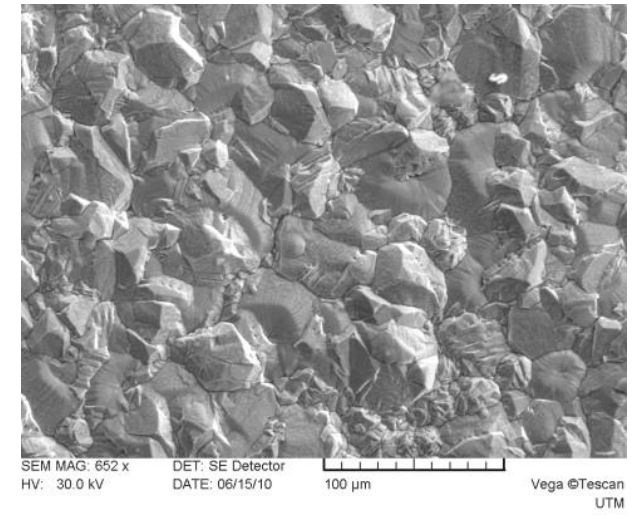

a view and thickness. Within the spots, numerous "filaments" of $0,2-0,3 \mathrm{mkm}$ in the diameter were revealed. These surfaces in some parts were covered with a "film", which made them smooth. The distinctive features of copper morphology (Fig. 6) differed substantially also with an increase in the deposition time $[11,12$, 14-18].

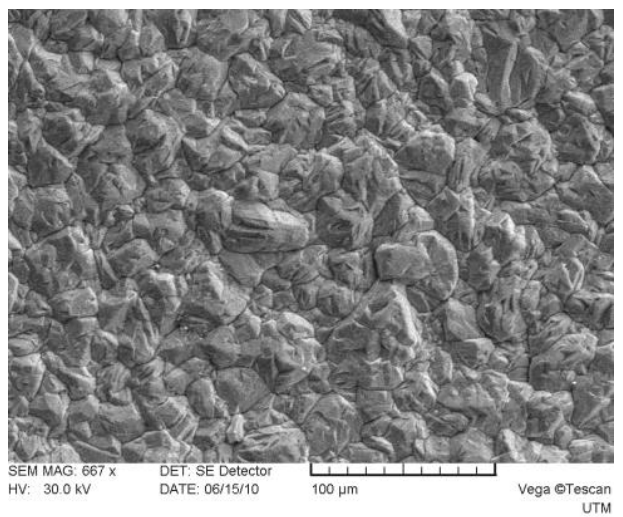

b

Fig6. Morphology of copper deposited from sulphate electrolyte (a single-phase PS) $\left(i_{k}=0,2 \mathrm{kA} / \mathrm{m}^{2}, i=100 \mathrm{~mA}\right.$, $\left.t_{e l}=20-22^{\circ} \mathrm{C}\right): a$ - without the ICD connection; $b$ - with the ICD connection $(L=10 \mathrm{H}, C=17600 \mathrm{mF})$.

The ICD parameters affect as well the structure of copper and nickel coatings deposited using various power sources [11, 12, 14-18].

As a rule at $\mathrm{L}_{\text {opt }}, \mathrm{C}_{\mathrm{opt}}$, the aggregates in the coatings decreased with a cathode form of the current remaining unchanged. The use of the switching power supply allowed us to a greater degree change the morphology and structure of the coatings, because in addition to the parallel connection of $\mathrm{L}$ and $\mathrm{C}$ we can use the pattern of connection in series of $\mathrm{L}$ and $\mathrm{C}$, or just $\mathrm{C}$. The structure of the coatings can also be effectively affected by the ICD, changing the electrolyte composition [10]. With a decrease in the concentration of copper sulphate to $50 \mathrm{~g} / \mathrm{l}$ and increase in sulphuric acid to $450 \mathrm{~g} / \mathrm{l}$ from a single-phase power supply, the coatings were not actually deposited. After the ICD connection, the coatings were plated and there were separate crystals on their surfaces, whose morphology contained nanosized elements (Fig. 7).

The X-ray diffraction of copper coatings obtained using a single-phase rectifier from sulphite electrolyte at a current density of $0,2 \mathrm{kA} / \mathrm{m}^{2}$ showed that with the ICD connection the axis of the texture changed from [100] to [111], which supports a substantial effect of the device parameters on the structure of the sediments $[8,19]$.

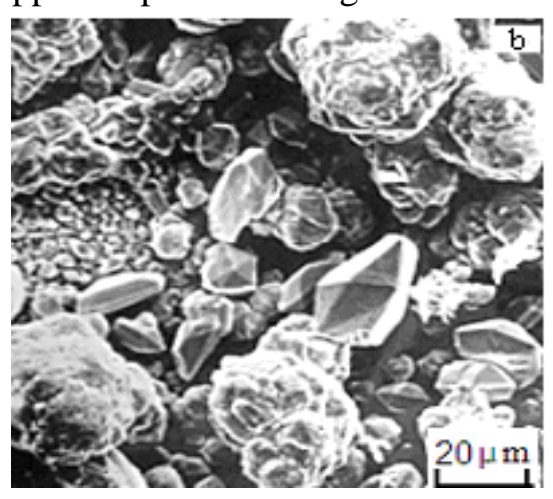

a

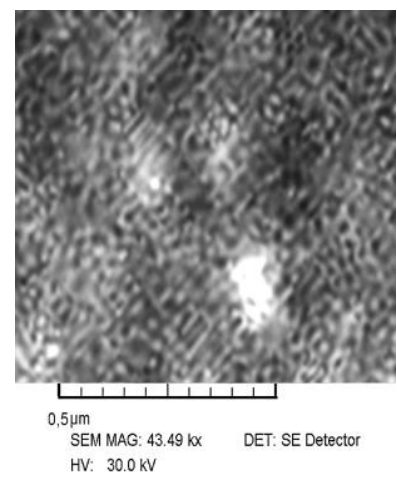

b

Fig7. Morphology of copper deposited from sulphate electrolyte (a single-phase $\mathrm{PS})\left(\mathrm{CuSO}_{4} \cdot 5 \mathrm{H}_{2} \mathrm{O}-50 \mathrm{~g} /\right.$, $\left.\mathrm{H}_{2} \mathrm{SO}_{4}-450 \mathrm{~g} / \mathrm{l}, i_{k}=0,2 \mathrm{kA} / \mathrm{m}^{2} ; i=100 \mathrm{~mA}, t_{e l}=20^{\circ} \mathrm{C}\right): a, b-$ with the ICD connection $(L=10 \mathrm{H}, \mathrm{C}=17600 \mathrm{mkF})$. 
The Dependence of Properties of the Electroplated Coatings on the Spectrum of the Variable Components of the Current (Voltage)

The ICD parameters affected substantially the morphology and structure of chromium coatings [18]. In the process of their deposition using a 3phase rectifier from a standard electrolyte and the ICD, we obtained bright coatings with a

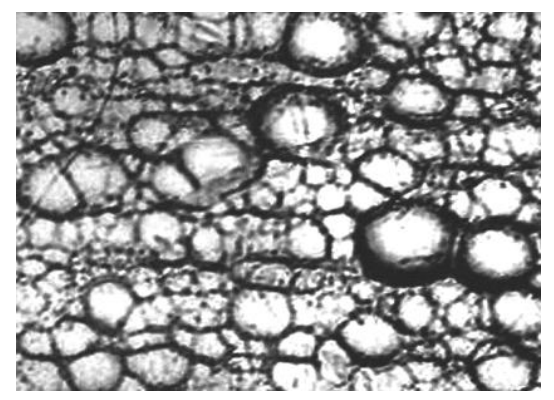

a current density increase to $12,0 \mathrm{kA} / \mathrm{m}^{2}$. Their morphology and structure differed markedly from the coatings developed under the optimal conditions of the electrolyte $\mathrm{i}_{\mathrm{k}}=5,5 \mathrm{kA} / \mathrm{m}^{2}$ without the ICD (Fig. 8, 9).

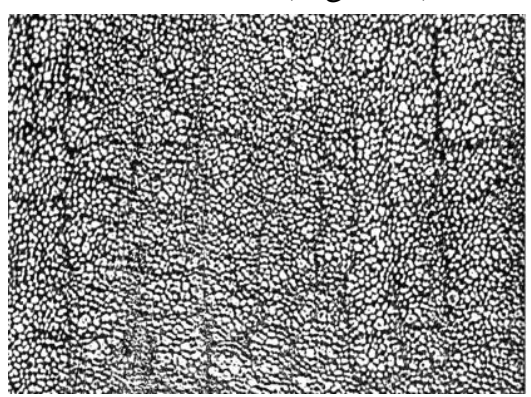

b

Fig8. Morphology of chromium coating from standard electrolyte (3-phase PS) $\left(i_{k}=8,5 \mathrm{kA} / \mathrm{m}^{2}, \mathrm{~h}=180 \mathrm{mkm}\right.$, x500): $a$ - without the ICD connection; $b$ - with the ICD connection $(L=0,119 \mathrm{mH}, C=0,024 \mathrm{~F})$.

The ICD makes it possible to increase the current density to $8.5-10.0 \mathrm{kA} / \mathrm{m}^{2}$ with micro hardness being increased to 11.0-12.0GPa, wear resistance increases by 2 times and the deposition rate from 30 $\mathrm{mkm} / \mathrm{h}$ to $85 \mathrm{mkm} / \mathrm{h}$.

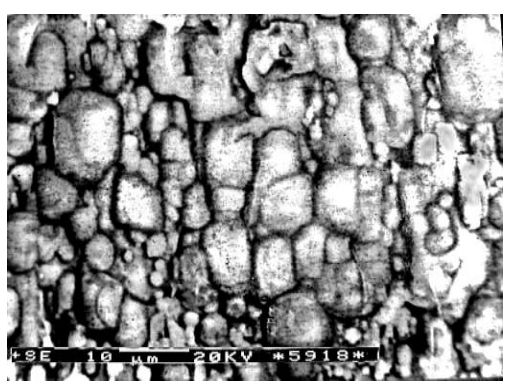

a

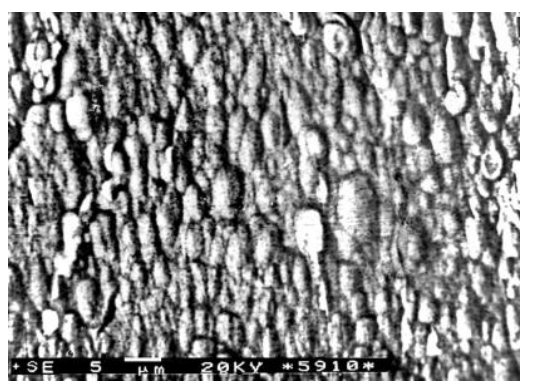

b

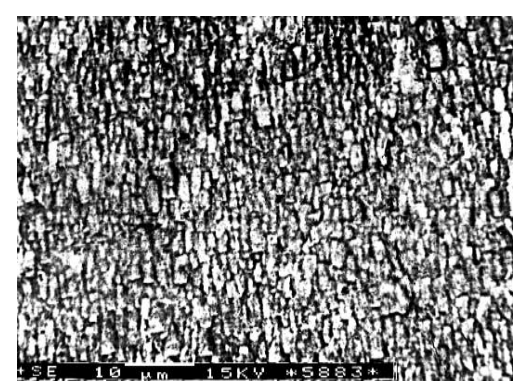

c

Fig9. Structure of chromium coating from a standard electrolyte (3-pase PS): a - without the ICD connection; $b$ - with the ICD connection $\left(i_{k}-5,5 \mathrm{kA} / \mathrm{m}^{2}, L=0,119 \mathrm{mH}, C=0,024 \mathrm{mF}\right) ; c$ - with the ICD connection $\left(i_{k}-8,5 \mathrm{kA} / \mathrm{m}^{2}, L=0,119 \mathrm{mH}, \mathrm{C}=0,024 \mathrm{mF}\right)$.

A similar effect the ICD produced also in the case of coating deposition using an accumulator as a power supply (Fig. 10).

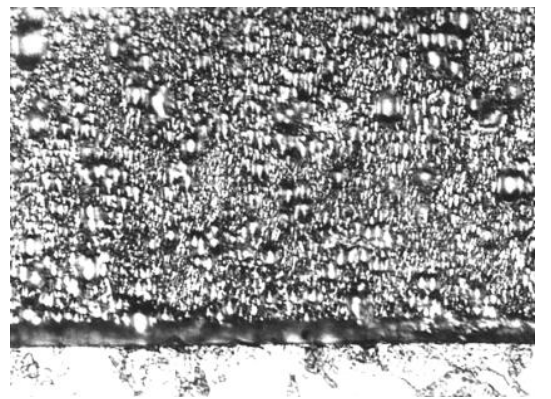

a

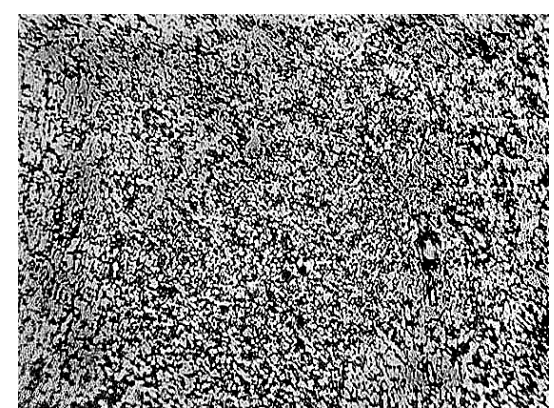

$\mathrm{b}$

Fig10. Structure of chromium coating from a standard electrolyte, accumulator PS: $\left(i_{k}=8,5 \mathrm{kA} / \mathrm{m}^{2}, x 500\right)$ : $a$ - without the ICD connection; $b$ - with the ICD connection $(L=0,09 \mathrm{mH}, C=0,01 \mathrm{~F})$.

An increase in microhardness from 5.00 to 8.00 GPa using the ICD was also registered in chromium coatings as well, which were developed from tetrachromic electrolyte $\mathrm{CrO}_{3}-$ $400 \mathrm{~g} / \mathrm{l}, \mathrm{NaOH}-60 \mathrm{~g} / \mathrm{l}, \mathrm{H}_{2} \mathrm{SO}_{4}-2,5 \mathrm{~g} / \mathrm{l}$, sugar $-1 \mathrm{~g} / \mathrm{l})$, which enhanced their wear resistance up to the level of the coatings deposited in the standard electrolyte without the ICD [13].

The efficiency of using the ICD was verified under the production conditions during the deposition of chromium, iron-nickel alloy, zinc and copper coatings. 
The Dependence of Properties of the Electroplated Coatings on the Spectrum of the Variable Components of the Current (Voltage)

\section{The Peculiarities of Deposition of Galvanic COATINGS USING THE INDUCNANCE - CAPACITANCE DEVICE}

In order to explain the effect of the variable components of the current on the process of deposition of coatings we studied the works devoted to the influence of magnetic and electric fields, as well as the overlapping of highfrequency currents on the main current [20-34].

During the deposition of coatings in the magnetic field, the change in the cathode polarization and the structure of the coatings can be attributed to the effect of the Lorentz force on the magnetohydrodynamic (MHD) convection $[20,21]$, which depends on the charge and the velocity of motion of ions in the electrolyte caused by the magnetic field intensity, as well as the location of the field lines on the electrode surface. The MHD convection increases the rate of the deposition, because in this case high concentration of ions is maintained near the electrode surface, which excludes both the diffusion limitations and an increase in the number of nucleation centers due to the decrease in the sizes of the diffusion zones (impoverishment zones) resulted from an increase in the mass transfer rate.

The electric field (EF) affects similarly the deposition process. The movement of liquid occurs as a result of the action of the electrodynamic force, which is connected with the density of the charges in the bulk or on the surface of the electrolyte. Microdynamic liquid flow in the region of a double layer is determined by the total effect of the action of the pulses of low molecular ions on the total liquid mass, i.e., it depends on the electric field induced by the electric charge of the surface interface and by the overall space charge in this region [32].

The electrode was rigidly attached to an areal, which was fed the pulses at a frequency of 30$3000 \mathrm{kHz}$ and excited mechanical oscillations (acoustic waves) in the solution. In this case, inequilibrium processes were put in order, and a statistical "noise" played the role of an automatic regulator of the signal. In the active medium with an internal power supply the nonlinear processes arise, and the wave crosscut makes the amplitude grow. The ultrasound effect can be connected with parametric resonance at certain frequencies when the acoustic wave increases the reactive parameters of the medium. If we accept that on the basis of the structures of a self-oscillatory type, first, self-organizing formations develop, which contain stable molecules, radicals and ions, with certain functional properties prevailing, then later they form synchronous stable groups, which react in the form of a resonance to the periodically controlled weak signal, and as a result of which a system of elements is created with abnormal kinetic properties of transfer into the reaction zone. This leads to an increase in the rate and energetic physicochemical processes and to the uniformity of the properties of products.

Overlapping of a high-frequency field ensures the formation of synergetic and coherent electrochemical and electrodynamics processes in electrolytes [29-33].

Coherence of the processes ensures the constancy of bonds between the phases, frequences, polarizations, amplitudes, as well as the formation of steady extended objects, which have a strictly ordered spatial arrangement. They appear inside the objects as a result of the electromagnetic field effect inside the electrolyte electron plasma due to which a strictly coherent motion of plasma microparticles occurs (electrons, ions, atoms and molecules) inside the object.

The presence in plasma of the volumetric macroscopic modes of charge transfer initiates the formation of a macroscopic current of the charge, in which a mechanism of resonance of currents is present at certain frequencies of the electromagnetic field, since the electric system has macroscopic properties of the capacity and inductance. The overlapping of the high frequency field also changes the electrolyte structure, because the presence of ions $\mathrm{H}^{+}$and $\mathrm{OH}^{-}$can establish hydrogen bonds among the typical radicals of the electrolyte which can occur more efficiently under the fulfillment the conditions of resonance-equality of the frequency of the field and frequency that characterises the hydrogen bond.

Taking into account that the cluster structure of the volume and of the near-cathode layer differ significantly, which makes it impossible to select a parametric mode to affect efficiently the volumetric and near-cathode processes, this points to the fact that the choice of frequency of the applied alternating current that influences the controlled parameters of the coating is also impossible, so the establishing of a compromise value of the current frequency becomes 
The Dependence of Properties of the Electroplated Coatings on the Spectrum of the Variable Components of the Current (Voltage)

necessary, which conventionally provides for the optimal characteristics of the galvanic coatings.

Therefore, the problem can be solved only at the presence of the spectrum of the variable components, whose values can be changed by varying the parameters ( $\mathrm{L}$ and $\mathrm{C}$ ) of the inductance-capacitance device, which is typical for the poly-frequency resonance.

\section{CONCLUSION}

Using the connection of the inductancecapacitance device to the power supply together with the varying of the values $L$ and $C$, makes it possible to affect the spectrum of the components of the current (voltage) which changes the kinetics of deposition and physicomechanical properties of the coatings. Obviously, in this case, the electrodynamic state is formed that induces the change in the hydrodynamic micromotion of the electrolyte in the near-cathode layer, as well as synchronizes the stages of the electrochemical process and exhibits the effect of resonance, due to which the deposition rate increases and the coatings'properties improve.

\section{REFERENCES}

[1] John L. Dow Son Electrochemical Woise Measurement. The Definitive In-Situ Technique for Corrosion Applications. J.R. Keerns, J.R. Scully, P.R. Roberge, D.L. Reichert, J.L. Dow Son Electrochemical Woise Measurement for corrosion applications. USA, STP, 1277 (1966), pp. 3-38.

[2] Fetter K., Electrochemical Kinetics. Moscow, 1964.

[3] Budevski E.B. Some Fundamental Aspects of Electrocrystalization //Progress in Surface and Membrane Science. 1976, v. II, 71-116.

[4] Tyagai V.A., Noises in the Electrochemical Systems Electrochemistry, 1973, vol. 10, 3-24.

[5] Kanevskii L.S., Grafov E.M., Study by the Method of Electrochemical Noises of the Dynamics of Passaging of Lithium Elrctrode in Aprotonic Organic Electrolytes. Electrochemistry, 3008, vol. 44, (5), pp. 515519.

[6] Gologan V.F., Control of Electrochemical Processes by Inductive-Capacitive Devices: Review - Surface Engineering and Applied Electrochemistry. 2005, 6, 36-40.

[7] G ologan V.F., Ungureanu V.M. Ustroistvo dlya electrochimicheskih processov. (Apparatus for electrochemical processes). Author's Certificate on the USSR N 1621559. Priority 15 September 1990.
[8] Gologan V.F., Bobanova Zh.I., Ivashku S.Ch. Dispozitiv pentru procese electrochimice (Apparatus for electrochemical processes). Author's Certificate on the Republic Moldova N MD 789. Priority 30.06.2014.

[9] Vagramyan A.T., Zhemargortsyants M., Electrodeposition of Metals and Inhibitory Adsorption. Moscow, 1969.

[10] Influence of the Composition of the Solution on the Process of Copper Deposition while using the Inductive-Capacitive Device. In: Electrolysis: Theory, Types and Applications. Nova Publishers (USA) 2010, 479-492.

[11] GologanV.F., Bobanova Zh.I., Ivashku S.Kh. On the Influence of the Parameters of Inductive-Capacitive Device on the Process of Electroplates Deposition Surface Engineering and Applied Electrochemistry. 2013, 49(3), 189-193.

[12] Gologan V.F., Bobanova Zh.I., Ivashku S.Kh. Peculiarities of Chromium Deposition with Application of an Induction-Capacitance Device Surface Engineering and Applied Electrochemistry. 2008, 44, (5), 353-358.

[13] Gologan V.F., Bobanova Zh.I., Enachi M.A., Ivaschu S.Kh., Croitoru D.M. Wear-resistant Chromium Coatings Deposited in Tetrachromate Electrolyte Connected to an Inductive-Capacitive Device. Surface Engineering and Applied Electrochemistry. 2014, 50(2), 111-116.

[14] Gologan V.F., Bobanova Zh.I., Ivashku S.Kh., Pasincovschi E.A. Effect of the Pulse Current and Capacitance Device on Copper Deposition. International Journal of Research Studies in Science, Engineering and Technology. ISS N 2349-475 (Print) and ISS N 2349-476X (Online). 2015, 2(7), 88-95.

[15] Gologan V.F., Bobanova Zh.I., Ivashku S.Kh. The Influence of a Power Source on an Induction-Capacitance of the Process of Nickel Deposition. Surface Engineering and Applied Electrochemistry. 2015, 5(3), 220 - 227.

[16] Gologan V.F., Bobanova Zh.I., Monaiko E., Mazur V., Ivashku S., Kiriyak E. Peculiarities of the Influence of an Inductance-Capacitance Device on the Initial Stage of the Crystallization of Electrolytic Coatings of Copper. Surface Engineering and Applied Electrochemistry. 2010, 46(1), 9-15.

[17] Gologan V.F., Bobanova Zh.I., Ivashku S.Kh., Volodina G., Mazur V., Pushkash B. Morphology of Electrolytic Copper Coating at Application of a Power Supply with an Induction-Capacitance Device Surface Engineering and Applied Electrochemistry. 2008. 43(1), 15-22.

[18] Gologan V.F., Bobanova Zh.I., Ivashku S.Kh., Influence of an Induction-Capacitance Device 
The Dependence of Properties of the Electroplated Coatings on the Spectrum of the Variable Components of the Current (Voltage)

on the Structure and wear Resistance of Electrolytic Chromium Coating. Surface Engineering and Applied Electrochemistry. 2008, 44(5), 353-358.

[19] Kochergin S.M., Leont'ev A.V., Texture Formation during Electrocrystallization of Metals. Moscow: Metallurgy, 1974.

[20] Matsushima H., Ispas A., Bund A., Plieth W., Fukunaka Y. Magnetic Field Effects on Micro structural Variation of Electrodeposited Cobalt Film. J. Solide State Electrochem. 2007, 11, 737-743.

[21] Coey J.M.D., Phen F.M.F., Dumne P., Murry S.M. The Magnetic Concentration Gradient Force - is it Real. J. Solide Stat Electrochem. 2007, 11, 711-717.

[22] Bondarenko N.F., Gak E.Z., Electromagnetic Phenomena in the Natural Waters. Leningrad: Hedrometeoizdat, 1981, $151 \mathrm{p}$.

[23] Kiseleva O.L., Kolesnikov A.A,, Zarembo V.I., et al., Increase in the Rates of Physicochemical Conversions in the Mode of Resonance Electromagnetoacoustic Transformation. Khim. Prom. 2003, 80(5), 12-24.

[24] Kiseleva O.L., Kolesnikov A.A,, Zarembo V.I., et al., Structuring of Inorganic Materials Using Weak Electromagnetic Fields in the Radio Frequency Range. Inorgan. Materials, 2004, 40(1), 96-102.

[25] Zarembo V.I., Kolesnikov L.L., The Background Resonance-Acoustic Control by Hetero-Phase Processes. Teor. Osnovy Khim. Tekhnologii. 2006, 40(5), 520-532.

[26] Zarembo V.I., Kolesnikov A.A., Ivanov E.V., The Background Electromagnetoacoustic Control by Structural and Plastic Properties of Metal materials, Izv. RAN, Ser. Fiz., 2006, 70(9), 1088-1091.
[27] Zarembo V.I., The Discharge of CopperMagnesium Galvanic Element in Weak Electromagnetic Field, Zh. Fiz. Khem. 2007, 81(7), 1339-1341.

[28] Kolesnikov A.A., Zarembo Ya.V., Puskov L.V., Zarembo V.I., The Electrochemical Recovery of Zinc on the Cathode in the Weak Electromagnetic Field, Zh. Fiz. Khem. 2007, 81(10), 1914-1916.

[29] Bramin V.A., Beznosyuk S.A., Mechanism of Coherency in Electrochemical and Electrodynamic Processes of Electrolytes, Izv. Altai Gos. Univ., Issue 2, 2005

[30] Kaplin A.A., Bramin V.A., Stas I.E., Inversive Voltammetry in High Frequency Electromagnetic Field, Zh. Analytic Khem., 1988, vol. 43, issue 4.

[31] Umedzova Kh., Matsumoto Kh., Tatiki M., Thermofield Dynamics and Condensed State, Trans from Eng., Moscow, 1985.

[32] Beznosyuk S.A., Quantum Reology and Confainment of Electrons in Nanostructures of the Condensed State, Izv. Vuzov, Fiz., 1994, 37(8).

[33] Khaken G., Synergetics, Moscow, 1980.

[34] Gologan V.F., Bobanova Zh.I., Bologa M.K. On the Role of Variable Components of the Current in the Deposition of Galvanic Coatings. International Journal of Research Studies in Science, Engineering and Technology (ISSN Print: 2349-4751, ISSN Online: 2349-476X) 2016, 3, (11), 6-10

Citation: V. F. Gologan, et al. "The Dependence Of Properties Of The Electroplated Coatings On The Spectrum Of The Variable Components Of The Current (Voltage)". International Journal Of Research Studies In Science, Engineering And Technology, vol 4, no. 5, 2017, pp. 1-9.

Copyright: (C) 2017 V.F. Gologan, et al. This is an open-access article distributed under the terms of the Creative Commons Attribution License, which permits unrestricted use, distribution, and reproduction in any medium, provided the original author and source are credited. 